\title{
Role of Colour Doppler in Evaluation of Hemodynamic Changes in Chronic Hepatitis C Infection
}

\author{
Mahjabeen Masood, ${ }^{1}$ Iqbal Hussain Dogar, ${ }^{2}$ Mahesh Gautam, ${ }^{3}$ Ghulam Rasul ${ }^{4}$
}

\begin{abstract}
Purpose of the Study: The estimation of the degree of hepatic fibrosis is important for diagnostic and therapeutic management of patients with chronic liver disease, in particular those associated with hepatic viral infections. In cirrhosis portal blood inflow, hepatic resistance and portal venous pressure increase. Doppler sonography is non-invasive diagnostic modality based on hemodynamic parameters. Hemodynamic changes might have developed even in cases with normal findings on B mode sonography. Therefore, assessment of these alterations has importance for early diagnosis and for close follow-up of previously dia-
\end{abstract}

Date of Submission 19-11-2015

Date of Revision Received 4-4-2016

Date of Acceptance for Publication 3-5-2016

Conflict of Interest: None

Funding Source: None

Masood M. ${ }^{1}$

Assistant Professor of Radiology

KEMU/ Mayo Hospital, Lahore

Dogar I.H. ${ }^{2}$

Associate Professor / HOD of Radiology

KEMU / Mayo Hospital, Lahore

Gautam M. ${ }^{3}$

Resident Department of Radiology, Mayo Hospital, Lahore

Rasul G. ${ }^{4}$

SMO, Department of Radiology, Children Hospital, Lahore gnosed case. Therefore the main aim of this study is to determine the role of colour flow Doppler ultrasound examination for detection of change in liver hemodynamic in hepatitis $\mathrm{C}$ positive chronic liver disease.

Materials and Methods: This study was conducted in Department of Radiology, Mayo Hospital Lahore from 2012 to 2013. Hundred diagnosed case of Hepatitis $\mathrm{C}$ by serology were included in this study. Patient were examined for liver and spleen size, texture and presence and absence of ascites. All the data were coded and analyzed using SPSS version 20.

Results: Mean age of the patient was found to be $45.90 \pm 12.71$ years. The echotexture of liver was normal in 29 patients $(29.0 \%)$, coarse with smooth margins in 48 patients $(48.0 \%)$ and coarse with irregular margins in 23 patients (23.0\%). Splenic size was enlarged $(\geq 13 \mathrm{~cm})$ in $63.0 \%$. Portal vein diameter was enlarged in $(\geq 13 \mathrm{~mm})$ in $53.0 \%$. Severity of ascites shows mild ascites in $24.0 \%$, moderate in $24.0 \%$ and severe in $19.0 \%$ patients. Portal vein velocity shows abnormality $(\geq 24 \mathrm{~cm} / \mathrm{sec})$ in $63.0 \%$. Hepatic vein waveform in $71.0 \%$ of patients was triphasic and nontriphasic in $29.0 \%$.

Conclusion: In conclusion alterations of liver hemodynamics resulting from inflammation at the tissue level are detectable in Doppler sonography.

Keywords: Hepatitis C, Cirrhosis, Doppler, Hepatic vein.

\section{Introduction}

Chronic liver hepatitis is a widespread disease world over that causes serious disorders such as cirrhosis and hepatocellular carcinoma. ${ }^{1}$ Despite its limited value sonography is still the most established method for the diagnosis and follow-up of chronic liver disease pri- 
marily because of its availability, low cost and noninterventional nature. Although a coarse echo pattern of liver and peril portal fibrosis may be detected, sonographic findings are normal in many cases. ${ }^{2}$ Cirrhosis has to be closely investigated because of its important complications, including ascites, peritonitis, portal hypertension and variceal bleeding.

Sonography is helpful in the diagnosis and follow up of the patients who have classic finding of the cirrhosis and portal hypertension such as ascites, varices or increasing portal vein diameter. However, B - mode sonography I incapable of examining cases with normal liver echogenicity and without any portal hypertension findings. ${ }^{3}$ Hepatic fibrosis is a known cause of several regional hepatic hemodynamic changes including the resistive index, hepatic blood flow, and the velocity of blood in the portal and hepatic arteries. ${ }^{4}$

In cirrhosis portal blood inflow, hepatic resistance and portal venous pressure increase. Despite the initial increase, portal blood inflow decreases with increasing sinusoidal resistance and development of portosystemic collateral vessels. ${ }^{5}$ The degree of liver dysfunction and portal hypertension are influenced by these changes in the hemodynamic of liver. Doppler ultrasound is a non-invasive modality by which theflow velocity and portal venous pressure as well as other parameters could be assessed. ${ }^{6}$ The changes in hemodynamic can be found in cases with normal grey scale findings. Thus for early diagnosis and follow-up of diagnosed case assessment of these alterations in hepatic blood flow is necessary. Therefore the main aim of this study is to determine the role of colour flow Doppler ultrasound examination for detection of change in liver hemodynamic in hepatitis $\mathrm{C}$ positive chronic liver disease.

\section{Methods}

This study was conducted in Department of Radiology, Mayo Hospital Lahore from 2009 to 2010. Hundred diagnosed case of Hepatitis $\mathrm{C}$ by serology were included in this study. History of present illness was obtained. Patient was examined for liver and spleen size, texture and presence and absence of ascites. The data were recorded in a preformed proforma. All the data were coded and analyzed using SPSS version 20.

\section{Results}

In this study, $63(63.0 \%)$ were males and 37 (37.0\%) were females. The maximum number of the patients $39(39 / 0 \%)$ were between $41-50$ years of age and 5 patients $(5.0 \%)$ belonged to age group of less than 20 years. The mean age was found to be $45.9 \pm 12.71$ years. Anti-HCV antibody test was positive in all patients $(100 \%)$.

The echotexture of liver was normal in 29 patients (29.0\%), coarse with smooth margins in 48 patients $(48.0 \%)$ and coarse with irregular margins in 23 patients $(23.0 \%)$. Splenic size was normal $(<13 \mathrm{~cm})$ in 37 patients $(37.0 \%)$ and was found enlarged $(\geq 13 \mathrm{~cm})$ in 63 patients $(63.0 \%)$. Portal vein diameter was normal $(<13 \mathrm{~mm})$ in 47 patients $(47.0 \%)$ and enlarged $(\geq 13 \mathrm{~mm})$ in 53 patients $(53.0 \%)$.

Minimal ascites (fluid in hepatorenal pouch) was found in 24 patients $(24.0 \%)$, moderate (fluid in pelvis) in 24 patients $(24.0 \%)$ and severe (both abdominopelvic ascites) in 19 patients (19.0\%). Ascites was absent in 15 patients $(15.0 \%)$.

Portal vein velocity was abnormal $(\geq 24 \mathrm{~cm} / \mathrm{s})$ in 63 patients $(63.0 \%)$. While remaining 37 patients $(37.0 \%)$ had normal portal vein velocity $(<24 \mathrm{~cm} / \mathrm{s})$.

Hepatic vein waveform shows 71 patients $(71.0 \%)$ of triphasic and 29 patients $(29.0 \%)$ belong to non triphasic.

\section{Discussion}

Chronic viral hepatitis is a widespread disease in the world that causes serious disorders such as cirrhosis and hepatocellular cancer. Despite its limited value, sonography is still the most established method diagnosis and follow-up of CVHprimarily because of its availability. Although coarse echo pattern of the liver and periportal fibrosis may be detected, sonographic findings are normal in any cases. Cirrhosis has to be closely investigated because of its important complications including ascites, peritonitis, portal hypertension and variceal bleeding. Sonography is helpful in the diagnosis and follow-up of the patient who have the classical findings of cirrhosis and portal hypertension such as ascites, varices or increasing portal vein diameter.

However, in B - mode sonography it is difficult to differentiate cases with normal liver echogenicity and without any portal hypertension findings. The various histologic component of chronic liver inflammation and repair could theoretically affect hepatic circulation by way of either dilation or constriction, through effects of inflammatory cytokines and anatomic impair- 
ment. Hepatic fibrosis is a known cause of several regional hepatic hemodynamics changes including the hepatic blood flow, resistive index, and the portal vein velocity. ${ }^{4}$

In cirrhosis, portal blood inflow, hepatic resistance and portal venous pressure increases. Despite the initial increase, portal blood inflow decreases with increasing sinusoidal resistance and development of portosystemic collateral vessels. ${ }^{5}$ The degree of liver dysfunction and portal hypertension are influenced by these changes in the hemodynamic of liver. Doppler ultrasound is a non-invasive modality by which the flow velocity and portal venous pressure as well as other parameters could be assessed. ${ }^{6}$ The changes in hemodynamic can be found in cases with normal grey scale findings. Thus for early diagnosis and follow-up of diagnosed case assessment of these alterations in hepatic blood flow is necessary.

Alterations of liver hemodynamics in $\mathrm{CVH}$ and cirrhosis have been observed in various studies. Some authors evaluated these changes for $\mathrm{CVH}$, some evaluated them for cirrhosis and some had evaluated for both. ${ }^{7}$ Limitations of different values from many simpler Doppler parameters of liver vasculature made observers use new indices for more reliable evaluations such as modified hepatic index (MHI) congestion index, arterioportal ratio and portal hypertension index. However, the reliability of these composite index is not proved yet. ${ }^{8}$

In this study the role of Doppler sonography in $\mathrm{HCV}$ patients was observed. Certain hemodynamic disruptions in liver vessels go along with chronic hepatic diseases. Pulsatility index, resistive index, hepatic blood flow, hepatic veins waveforms, portal vein velocity and hepatic arteries parameters are susceptible to morphologic and pathologic changes.

These parameters can reliably be evaluated by Doppler sonography. Many reports confirmed the clinical usefulness of this technique in the diagnosis of the portal hypertension and liver cirrhosis. ${ }^{9}$ Cirrhosis is characterized by the presence of the extensive fibrosis and numerous regenerative nodules replacing the normal liver parenchyma. Elevated vascular resistance and increased portal blood inflow are two principle mechanisms that have been reported to be involved in the development of portal hypertension secondary to cirrhosis. ${ }^{10}$

In this study portal vein diameter. Portal vein velocity, PVP score and hepatic vein waveform were studied using duplex ultrasound Doppler (GE Logic 5 machine). The result of this study support the results of Puneet et al. who also found that the PVV correlates with the clinical stage of the disease. ${ }^{11}$ Contrary to this, Shateri et al, found no significant correlation between portal flow velocity and liver fibrosis. ${ }^{12}$

In healthy subjects hepatic vein waveform is triphasic. Due to the changes in liver parenchyma, the compliance of hepatic vein is impaired. Stiffness of liver parenchyma in chronic hepatitis results in dampening of the waveform. Only few studies are available regarding the relationship between non triphasic flow and histopathological changes.The increase in the frequency of non-triphasic flow with more severe fibrosis is not in contradiction with a report by Thomas et al, who concluded that loss of normal triphasic flow is highly sensitive in predicting varices in cirrhotic patients. ${ }^{13}$ Sudhamshu et al, also suggested that the abnormality of hepatic vein waveforms correlate with steatosis andfibrosis in the adjacent liver parenchyma. ${ }^{14}$ The alteration of the hepatic vein waveform was also noted with hepatic steatosis. Nontriphasic flow was appreciated in patients with increased liver echogenicity. ${ }^{15}$ This result is also in concordant with previous observations.

Although HARI and HAPI is not calculated in this study, some international studies suggests that these parameters were significantly greater in patients with moderate or severe inflammation than those with mild inflammation in controlled studies. Although only few study regarding HARI and HAPI values are observed in CVH patients. ${ }^{16}$ Piscaglia et al, concluded that HARI in patients with CVH seems to be significantly influenced by the degree of fibrous tissue deposits. ${ }^{17} \mathrm{It}$ is important to disclose the increase in HARI and HAPI in mildly inflamed cases of CVH. Once the inflammatory degree exceeds the mild phase, the increase in the hepatic artery resistance varies dependent of inflammation. This means that hepatic arterial resistance indices in moderate inflammation can be higher than those in severe inflammation.

The relationship between the portal and splenic vein diameters, liver and spleen sizes as well as Doppler parameters of afferent vasculature and collaterals were observed. The results were not helpful in assessing the severity of chronic liver disease or cirrhosis. This results were parallel with those of the previous studies. ${ }^{18}$ No relationship was found between cases with and without ascites for Doppler parameters of afferent vasculature, portal and splenic vein diameters or spleen size in various international studies. ${ }^{18}$

Studies suggesting the significant differences of PVV between CVH and cirrhosis are limited. Signifi- 
cant differences of PVV, portal and splenic vein diameters and spleen size was found between the $\mathrm{CVH}$ and cirrhotic groups. PVV was significantly lower in cirrhotic groups, whereas the others were significantly lower in the $\mathrm{CVH}$ groups.

\section{Conclusion}

In conclusion alterations of liver hemodynamics resulting from inflammation at the tissue level are detectable in Doppler sonography. This noninvasive technique is helpful in assessing the degree of the liver inflammation and the stage of fibrosis, and it should be used to follow the progression of CVH. Doppler sonography has high diagnostic value for cirrhosis. However, it value in clinical staging is limited.

\section{References}

1. Liberek A, Szaflarska - Poplawska A, Luczak G, GoraGebka M, Jankowska A, Los-Rycharskda E, et al. [Therapy of chronic viral hepatitis type $B$ in children after previous ineffective interferon-alpha treatment]. Medycyna wieku rozwojowego. 2007; 11 (4): 373-9.

2. Iliopoulos P, Vlychou M, Margaritis V, Tsamis I, Tepetes K, Petsas T, et al. Gray and color Doppler ultrasonography in differentiation between chronic viral hepatitis and compensated early stage cirrhosis. Journal of gastrointestinal and liver diseases : JGLD. 2007; 16 (3): 279-86.

3. Iagoda AB, Koroi PV, Geivandova NI, Nikitina OA, Kastornaia IV. [Growth factors and the hystologic picture of the liver in chronic viral hepatitis and hepatic cirrhosis]. Klinicheskaia meditsina. 2006; 84 (8): 44-7.

4. Mao Y-M, Zeng M-D, Lu L-G, Wan M-B, Li C-Z, Chen $\mathrm{C}-\mathrm{W}$, et al. Capsule oxymatrine in treatment of hepatic fibrosis due to chronic viral hepatitis: a randomized, double blind, placebo - controlled, multicenter clinical study. World Journal of Gastroenterology, 2004; 10 (22): 3269-73.

5. Haktanir A, Cihan BS, Çelenk Ç, Cihan Ş. Value of Doppler sonography in assessing the progression of chronic viral hepatitis and in the diagnosis and grading of cirrhosis. Journal of ultrasound in medicine, 2005; 24 (3): 311-21.

6. Lim AK, Patel N, Eckersley RJ, Kuo Y-T, Goldin RD, Thomas HC, et al. Can Doppler sonography grade the severity of hepatitis C-related liver disease? American Journal of Roentgenology, 2005; 184 (6): 1848-53.
7. La Villa G, Gentilini P. Hemodynamic alterations in liver cirrhosis. Molecular aspects of medicine, 2008; 29 (1-2): 112-8.

8. Popov D, Krasteva R, Ivanova R, Mateva L, Krastev Z. Doppler parameters of hepatic and renal hemodynamics in patients with liver cirrhosis. International journal of nephrology, 2012; 2012.

9. Gerstenmaier J, Gibson R. Ultrasound in chronic liver disease. Insights into imaging, 2014; 5 (4): 441-55.

10. Martell M, Coll M, Ezkurdia N, Raurell I, Genescà J. Physiopathology of splanchnic vasodilation in portal hypertension. World journal of hepatology, 2010; 2 (6): 208.

11. Mittal P, Gupta R, Mittal G, Kalia V. Association between portal vein color Doppler findings and the severity of disease in cirrhotic patients with portal hypertension. Iranian Journal of Radiology, 2011; 8 (4): 211.

12. Shateri K, Mohammadi A, Moloudi F, Nosair E, Ghasemi - rad M. Correlation Between Sonographic Portal Vein Diameter and Flow Velocity With the Clinical Scoring Systems MELD and CTP in Cirrhotic Patients: Is There a Relationship? Gastroenterology Research, 2012; 5 (3): 112-9.

13. Joseph T, Madhavan M, Devadas K, Ramakrishnannair VK. Doppler assessment of hepatic venous waves for predicting large varices in cirrhotic patients. Saudi journal of gastroenterology: official journal of the Saudi Gastroenterology Association, 2011; 17 (1): 36.

14. Sudhamshu K, Sharma D, Chataut SP. Hepatic vein waveforms in liver cirrhosis re-evaluated. Hepatology international, 2011; 5 (1): 581-5.

15. Gaiani S, Avogaro A, Bombonato G, Bolognesi M, Amor F, de Kreutzenberg SV, et al. Nonalcoholic fatty liver disease (NAFLD) in nonobese patients with diabetes: Prevalence and relationships with hemodynamic alterations detected with Doppler sonography. Journal of ultrasound, 2009; 12 (1): 1-5.

16. Pierce ME, Sewell R. Identification of hepatic cirrhosis by duplex Doppler ultrasound value of the hepatic artery resistive index. Australasian radiology, 1990; 34 (4): 331-3.

17. Piscaglia F, Zironi G, Gaiani S, Mazziotti A, Cavallari A, Gramantieri L, et al. Systemic and splanchnic hemodynamic changes after liver transplantation for cirrhosis: A long - term prospective study. Hepatology, 1999; 30 (1): 58-64.

18. O'Donohue J, Ng C, Catnach S, Farrant P, Williams R. Diagnostic value of Doppler assessment of the hepatic and portal vessels and ultrasound of the spleen in liver disease. European journal of gastroenterology and hepatology, 2004; 16 (2): 147-55. 